\title{
EGCG-S Impacts Oxidative Stress and Infection of Enterovirus 69 in Lung Cells
}

\author{
Hager Mohamed, Lee H. Lee, Sandra D. Adams* \\ Department of Biology, Montclair State University, Montclair, NJ, USA \\ Email:^adamssa@montclair.edu
}

How to cite this paper: Mohamed, H., Lee, L.H. and Adams, S.D. (2021) EGCG-S Impacts Oxidative Stress and Infection of Enterovirus 69 in Lung Cells. Advances in Bioscience and Biotechnology, 12, 109-124. https://doi.org/10.4236/abb.2021.125008

Received: May 4, 2021

Accepted: May 25, 2021

Published: May 28, 2021

Copyright (อ 2021 by author(s) and Scientific Research Publishing Inc. This work is licensed under the Creative Commons Attribution International License (CC BY 4.0).

http://creativecommons.org/licenses/by/4.0/

(c) (i) Open Access

\begin{abstract}
Enteroviruses are responsible for emerging diseases which cause diverse symptoms and may result in neurological complications. An antiviral with multiple mechanisms of action can help prevent enterovirus mediated disease despite differences in the pathogenesis between enteroviruses, including the recently identified enterovirus 69 (EV-69) for which pathogenesis is not well understood. This study investigated the efficacy of epigallocatechin-3-gallate stearate (EGCG-S), a modified form of the antioxidant green tea catechin epigallocatechin-3-gallate (EGCG), in inhibiting EV-69 infection of lung fibroblast cells in vitro. Treatment with EGCG-S resulted in moderate protection from EV-69 mediated cytotoxicity as demonstrated by increased metabolic activity as well as maintenance of cell morphology and mitochondrial function. These effects were correlated with reduced hydrogen peroxide production in infected cells following EGCG-S treatment with concentrations less than $100 \mu \mathrm{M}$, suggesting a role for inhibition of EV-69 mediated oxidative stress. This study provides insight into characteristics of EV-69 infection as well as the efficacy of EGCG-S mediated inhibition of EV-69 infection.
\end{abstract}

\section{Keywords}

ROS, Enterovirus 69, Picornavirus, Green Tea Flavanol, Antioxidant, Antiviral, MRC-5 Cells, A549 Cells

\section{Introduction}

Enteroviruses comprise a large and diverse genus of non-enveloped RNA viruses in the Picornaviridae family. Members of this genus are grouped into fifteen species all of which are associated with a wide variety of diseases due to their tropism encompassing gastrointestinal, respiratory, and neuronal cells [1]. This allows enterovirus infections affecting the gastrointestinal or respiratory tract to 
also cause neuropathogenesis and fatal conditions such as meningitis [2] [3].

The rapid mutation rate of enteroviruses allows for emergence of strains capable of causing severe disease and even within the same species of enteroviruses pathogenesis can greatly vary. The Human enterovirus D species, for example, includes enterovirus 68 (EV-68) which causes respiratory disease and acute flaccid paralysis while another serotype, EV-70, is primarily associated with acute hemorrhagic conjunctivitis [4] [5]. These serotypes are two of four enteroviruses most recently isolated, along with EV-69 and EV-71. EV-71 causes hand, foot, and mouth disease [6]. Both EV-68 and EV-70 along with EV-71, have been associated with severe neurological disease [7] [8] [9]. Much about the pathogenesis of EV-69, however, is still not known since its first reported isolation in the U.S. in 1959 [10]. This type was originally associated with respiratory illness similar to EV-68 [11] but in recent years has been isolated worldwide from patients presenting with acute flaccid paralysis and encephalitis [12]-[18]. While some common features of the enterovirus infectious cycle likely apply to EV-69, there is a lack of knowledge on the tissue tropism of this enterovirus serotype. This and other emerging enteroviruses may continue to cause outbreaks and disease associated mortality, necessitating novel antivirals that can alleviate infection regardless of the enterovirus type. Such antivirals would thus ideally be able to simultaneously target multiple stages of the infectious cycle to account for differences, such as different host receptor utilization, between enteroviruses.

Here, we investigate the modulation of oxidative stress and the therapeutic properties of a compound derived from the Camellia sinensis plant, known as epigallocatechin-3-gallate (EGCG). EGCG is a green tea polyphenol which has been applied to numerous infectious disease models for the inhibition of microbial growth and viral infectivity [19] [20] [21]. The antiviral effect of EGCG has been reported for both enveloped and non-enveloped viruses including the enteroviruses Coxsackie B3 and EV-71 [21]-[26]. Additionally, it has been demonstrated to be well tolerated in vivo and even utilized for alleviating neurotoxicity associated viral proteins in mice [20] [27]. While the mechanism behind EGCG mediated antiviral effects has been often proposed to be due to interfering with virus attachment to host cells [28], some studies have found that it is the antioxidant property and ability of EGCG to modulate cellular redox that is associated with inhibition of infection [26] [29] [30] [31]. The ability of EGCG to inhibit viral infection through various ways shows promise for the development of an antiviral agent that can be applicable to emerging viruses for which pathogenesis is still unknown. Furthermore, modifications have been made to the structure of EGCG to enhance its stability and bioavailability [23] [32]. Such include palmitoylation of EGCG (pEGCG), which resulted in more effective inhibition of HSV-1 adsorption and infection in Vero cells than did EGCG [23]. Furthermore, addition of stearic acid to EGCG to make epigallocatechin-3-gallate-stearate (EGCG-S) is another modification that enhanced stability and was also shown to inhibit HSV-1 infection in A549 cells without causing cytotoxicity at up to 75 $\mu \mathrm{M}$, similar to EGCG [33]. In this study, we investigate the cytoprotective effects 
of EGCG-S on infected cells and evaluate the efficacy of EGCG-S in inhibiting infection of EV-69 in MRC-5 cells and A549 cells. MRC-5 and A549 lung fibroblast cells are appropriate models for in vitro study because EV-69 is associated with atypical respiratory illness.

\section{Materials and Methods}

\section{Cell Culture Maintenance}

The adherent human lung epithelial A549 cells (CCL-185) and the fetal lung fibroblast IRR-MRC-5 cells (ATCC 55-X) (American Type Culture Collection (ATCC) Manassas, VA) were maintained in T25 flasks at $37^{\circ} \mathrm{C}$ in a $5 \% \mathrm{CO}_{2}$ incubator. The A549 cell line and MRC-5 cell line were propagated in $\mathrm{F} 12 \mathrm{~K}$ and MEM media (Gibco, ThermoFisher Scientific), respectively, both of which were supplemented with $10 \%$ Fetal Bovine Serum and 1\% gentamicin.

\section{Virus Propagation}

Cells were sub-cultured into a T25 flask and incubated $24 \mathrm{hrs}$ to reach $70 \%$ $80 \%$ confluency. $100 \mu \mathrm{L}$ of Enterovirus type 69 [American Type Culture Collection (ATCC ${ }^{\otimes}$ VR-1077 ${ }^{\mathrm{TM}}$ ) Manassas, VA] was used to infect the confluent cell monolayer for $1 \mathrm{hr}$ after which $5 \mathrm{~mL}$ of media was supplemented. MRC-5 cells were checked after $24 \mathrm{hrs}$ and A549 cells were checked after 48 - $72 \mathrm{hrs}$ for cytopathic effect (CPE). Virus was harvested when at least $90 \%$ of cells showed CPE, through removal of the media and centrifugation for $5 \mathrm{~min}$ at 1500 RPM to eliminate cellular debris. The supernatant containing virus was stored in cryogenic tubes at $-80^{\circ} \mathrm{C}$ and used for the following experiments.

\section{EGCG-S Preparation}

EGCG-stearate (EGCG-S) from green tea extract in powder form (US Patent 20120172423) was obtained from Camellix, LLC (Georgia Health Sciences University Center of Innovation for Life Sciences, August, GA). It was dissolved in DMSO to a final stock concentration of $5 \mathrm{mM}$ and stored at $4^{\circ} \mathrm{C}$. Treatment concentrations were prepared from this stock by diluting in F12K or DMEM to make $25,50,75$, and $100 \mu \mathrm{M}$ concentrations.

\section{MTS cell viability assay}

The CellTiter 96Aqueous One Solution Cell Proliferation Assay (MTS) kit (Cat\#G3580, Promega Corp., Madison, WI) was used to quantify cell viability. A549 and MRC-5 cells were seeded from a 100\% confluent flask into a 96-well plate and incubated for $24 \mathrm{hrs}$ at $37^{\circ} \mathrm{C}$ under $5 \% \mathrm{CO}_{2}$ prior to performing the assay. Cells at $70 \%-80 \%$ confluency were then treated with $100 \mu \mathrm{L}$ of $25,50,75$, or $100 \mu \mathrm{M}$ of EGCG-S for $1 \mathrm{hr}$. The unadsorbed EGCG-S was then aspirated and cells were supplemented with medium and incubated for $24 \mathrm{hrs}$ at $37^{\circ} \mathrm{C}$ under $5 \% \mathrm{CO}_{2}$. After incubation, $20 \mu \mathrm{L}$ of MTS reagent was added to each well and the plate was incubated for $1 \mathrm{hr}$. Absorbance was read at $490 \mathrm{~nm}$ using an Infinite Pro 200 microplate reader (Tecan Life Sciences). The percent viability was determined for each sample by first normalizing absorbance values according to the untreated cells using Microsoft Excel (Microsoft Office, New York, NY, 
USA), following the formula below. The Mean and Standard error of mean (SEM) of three to five replicates were then calculated using Prism 9 software (GraphPad, San Diego, CA, USA).

$\%$ Viability $=(($ Treated cells - Blank $) /($ Cells only - Blank $)) \times 100 \%$.

\section{MTS antiviral cell viability assay}

MRC-5 or A549 cells were seeded in a 96-well plate and incubated for $24 \mathrm{hrs}$ at $37^{\circ} \mathrm{C}$ in a humidified $\mathrm{CO}_{2}$ atmosphere until $80 \%-90 \%$ confluent. Virus was treated with 25, 50, 75, and $100 \mu \mathrm{M}$ EGCG-S for $1 \mathrm{hr}$ prior to infection. Cells were then infected with $100 \mu \mathrm{L}$ EGCG-S treated and untreated virus, respectively, for $1 \mathrm{hr}$ after which unadsorbed virus was aspirated and cells were replenished with media. The plate was incubated for $24 \mathrm{hrs}$ at $37^{\circ} \mathrm{C}$ in a humidified $\mathrm{CO}_{2}$ atmosphere, at which point $20 \mu \mathrm{L}$ of the MTS reagent was added and absorbance was read at $490 \mathrm{~nm}$ using a 96-well plate reader spectrophotometer following $1 \mathrm{hr}$ of incubation with the reagent. The \% Inhibition was calculated with Microsoft Excel using the formula below for each replicate absorbance value of each condition. Mean and Standard error of mean (SEM) for three to five replicates per experiment were then calculated using Prism 9 Software (GraphPad, San Diego, CA, USA).

$\%$ Inhibition $=[($ Cells infected with EGCG-S treated virus - Untreated infected cells $) /($ Untreated uninfected cells - Untreated infected cells $)] \times 100$.

\section{ToxGlo ATP assay}

A 96-well plate was plated with MRC-5 cells and incubated for $24 \mathrm{hrs}$ at $37^{\circ} \mathrm{C}$ in a humidified $\mathrm{CO}_{2}$ atmosphere until $80 \%-90 \%$ confluent. For infection assays, virus was treated with $25,50,75$, and $100 \mu$ M EGCG-S for $1 \mathrm{hr}$ prior to infection. Media was aspirated from wells and cells were then infected with $100 \mu \mathrm{L}$ EGCG-S treated and untreated virus for $1 \mathrm{hr}$. After infection, unadsorbed virus was aspirated and cells were replenished with media. The Viral ToxGlo ${ }^{\mathrm{TM}}$ assay kit (Cat\#G8941, Promega Corp., Madison, WI) was used to detect viable cells with normal mitochondrial function by measuring ATP levels in test conditions. The plate was incubated for $24 \mathrm{hrs}$ at $37^{\circ} \mathrm{C}$ in a humidified $\mathrm{CO}_{2}$ atmosphere, at which point $10 \mu \mathrm{L}$ of the ATP detection reagent was added to each well and the plate was incubated for $1 \mathrm{hr}$. Relative luminescence units (RLU) were then read as a correlate of ATP levels using a Biotek Synergy ${ }^{\mathrm{TM}} 2$ luminometer.

\section{Antiviral ROS-Glo $\mathrm{H}_{2} \mathrm{O}_{2}$ assay}

The ROS-Glo ${ }^{\mathrm{TM}} \mathrm{H}_{2} \mathrm{O}_{2}$ assay kit (Promega Corp., Madison, WI) was used to assess oxidative stress induced by viral infection by measuring the levels of $\mathrm{H}_{2} \mathrm{O}_{2}$, a reactive oxygen species (ROS). MRC-5 cells were seeded in a 96-well plate and allowed to reach $80 \%$ confluency, then infected with EGCG-S treated or untreated virus for $1 \mathrm{hr}$ as described in the previous infection assays. The lytic assay protocol for product Cat\#G8820 was followed as per manufacturer's directions. Cells were then incubated at $37^{\circ} \mathrm{C}$ in a humidified $\mathrm{CO}_{2}$ atmosphere for $7 \mathrm{hrs}$ to allow infection, after which $20 \mu \mathrm{L}$ of $\mathrm{H}_{2} \mathrm{O}_{2}$ substrate solution was added to each well and cells were incubated for an additional $5 \mathrm{hrs}$, for a total of $12 \mathrm{hrs}$ of in- 
fection. After incubation, $100 \mu \mathrm{L}$ of the ROS-Glo ${ }^{\mathrm{TM}}$ detection solution reaction was added to each well and the plate was incubated for 20 mins. ROS levels were measured in RLU and read using a Biotek Synergy ${ }^{\mathrm{TM}} 2$ luminometer.

\section{Determination of cytopathic effects in cells with EGCG-S treated virus}

MRC-5 and A549 cells were seeded in a 6-well plate and incubated for $24 \mathrm{hrs}$ until $70 \%-80 \%$ confluency. Virus was treated with $25,50,75$, or $100 \mu \mathrm{M}$ EGCG-S for $1 \mathrm{hr}$ prior to infection. Media was aspirated from adherent cells in each well and cells were then infected with $100 \mu \mathrm{L}$ EGCG-S treated and untreated virus, respectively, for $1 \mathrm{hr}$ after which unadsorbed virus was aspirated and cells were replenished with media. The plate was incubated at $37^{\circ} \mathrm{C}$ under $5 \% \mathrm{CO}_{2}$. Cells were examined for $\mathrm{CPE}$ at $400 \times$ using an inverted microscope and images were taken at 48 and 72 hrs post infection (hpi).

\section{Statistical Analysis}

Graphing and statistical analysis was done using Prism 9 Software (GraphPad, San Diego, CA, USA). Data was analyzed using a one-way Anova with Dunnett's post-hoc test unless otherwise mentioned.

\section{Results}

\subsection{Cytotoxicity Study of Treatment of A549 and MRC-5 Cells with EGCG-S}

Enteroviruses are able to infect a variety of cells which include cells of the respiratory tract and can be found in respiratory secretions [34] [35]. To understand the potential effect of EGCG-S treatment on EV-69 infection in cells of the respiratory tract, A549 lung epithelial cells and MRC-5 lung fibroblast cells which were previously demonstrated to be highly susceptible to enterovirus infection were used [36]. Cytotoxicity to EGCG-S was assayed at a concentration range of $25-100 \mu \mathrm{M}$. Since EGCG-S was dissolved in DMSO, the effect of DMSO alone on cells was evaluated. Microscopy analysis showed that cells were not affected by the DMSO vehicle control even when treated at concentrations greater than $0.5 \%$ (The highest final concentration of DMSO used in the study). This correlated well with a previously reported study [37]. Cell viability of A549 cells and MRC-5 cells treated with 25 - $100 \mu$ M EGCG-S was determined using an MTS assay, which quantifies viable cells through detection of cellular respiration using the insoluble formazan product as an indicator. This formazan product is derived from the MTS substrate (3-(4, 5-dimethylthiazol-2-yl)-5-(3-carboxymethoxyphenyl)-2-(4-sulfophenyl)-2H-tetr azolium, inner salt) which becomes converted by the mitochondrial dehydrogenase enzymes present in viable cells. Cytotoxicity did not occur with EGCG-S treatment at up to $75 \mu \mathrm{M}$ (Figure 1(a)) for A549 cells and no cytotoxicity was observed for MRC-5 cells at each concentration used $(25,50,75$, and $100 \mu \mathrm{M})$ (Figure 1(b)). Treatment with these concentrations even resulted in a slightly higher cell viability relative to the untreated control for MRC-5 cells, possibly as a result of antioxidant or protective effects of EGCG-S against regular cell 
A549

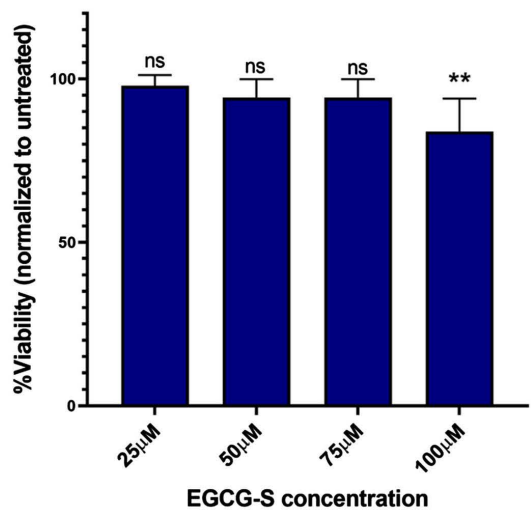

(a)
MRC-5

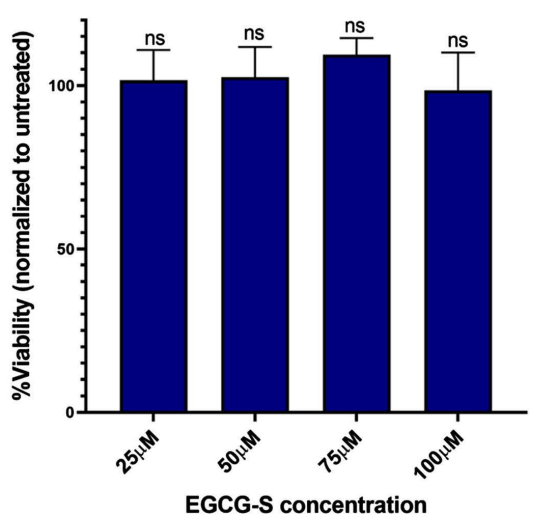

(b)

Figure 1. EGCG-S is non-toxic to A549 and MRC-5 cells up to $75 \mu \mathrm{M}$. A549 cells $(\mathrm{n}=5)$ and MRC-5 cells $(n=3)$ were cultured in 96-well plates and treated 24 hrs later with 25 , 50, 75 or $100 \mu \mathrm{M}$ of EGCG-S for $1 \mathrm{hr}$. The \% viability was determined using the CellTiter 96Aqueous One Solution Cell Proliferation Assay (MTS) (Promega). A549 (a) and MRC-5 cells (b) remain viable with EGCG-S treatment relative to the untreated control at up to $75 \mu \mathrm{M}$ or $100 \mu \mathrm{M}$, respectively. Data are presented as mean $\pm \mathrm{SEM},{ }^{* *} \mathrm{p}<0.01$, ns $=$ non-significant.

damage pathways. The cytotoxicity of EGCG-S on cells is dose dependent. EGCG-S was safely applied to cultured A549 cells [33] and cultured Vero cells (unpublished data) up to $75 \mu \mathrm{M}$. The results indicated that EGCG-S is non-cytotoxic to A549 and MRC- 5 cells.

\subsection{Proliferation Assay of A549 and MRC-5 Cells Infected with EV-69 and EGCG-S Treated EV-69}

The ability of EGCG-S to protect against EV-69 mediated cytotoxicity was further investigated by measuring viability with respect to metabolically active cells as well as ATP levels as an indicator of normal mitochondrial function. The percent inhibition of infection was determined based on these viabilities as described in the Methods section. An increased viability of cells with treated EV-69 as compared to the untreated EV-69 was observed (Figure 2(a)). The highest inhibition of infection observed was $47 \%$ after $75 \mu \mathrm{M}$ treatment for A549 cells (Figure 2(b)). The percent inhibition of infection was overall lower for MRC-5 cells at the same EGCG-S concentrations tested for A549 cells, at most being $24.5 \%$ after $50 \mu \mathrm{M}$ treatment (Figure 2(b)). Altogether, this demonstrates that EGCG-S treatment has some efficacy in inhibiting EV-69 infection but highlights the differences in efficacy of EGCG-S in vitro that can be expected between different cells of the respiratory tract infected with EV-69. Infection with EGCG-S treated EV-69 results in increased cell viability of both A549 and MRC-5 cells compared with untreated virus infected cells. Since the A549 cells failed to support productive EV-69 infections, the extent of EGCG-S mediated protection against the cytotoxic effects of EV-69 infection of the MRC- 5 cells was further investigated. 


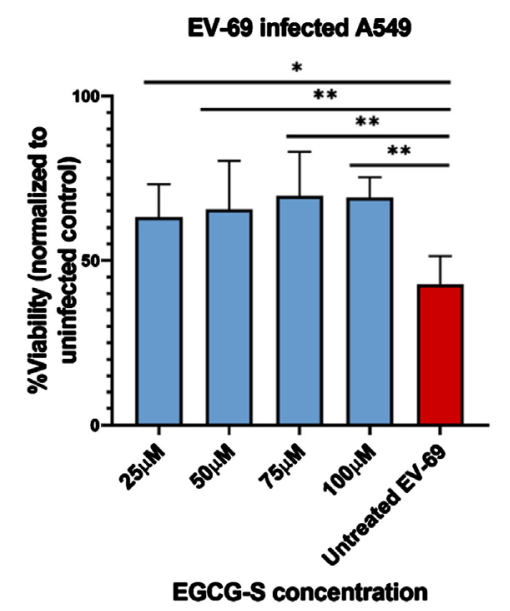

EV-69 infected A549

GCG-S concentration

A549

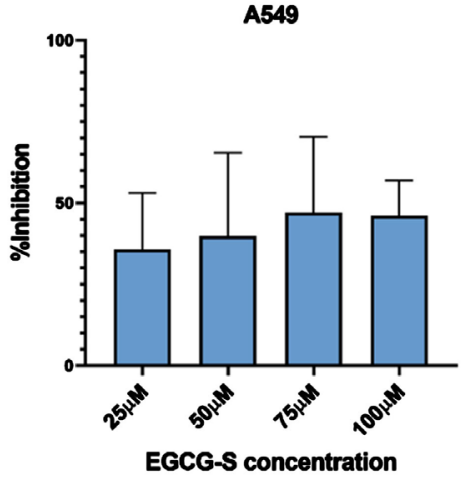

EV-69 infected MRC-5

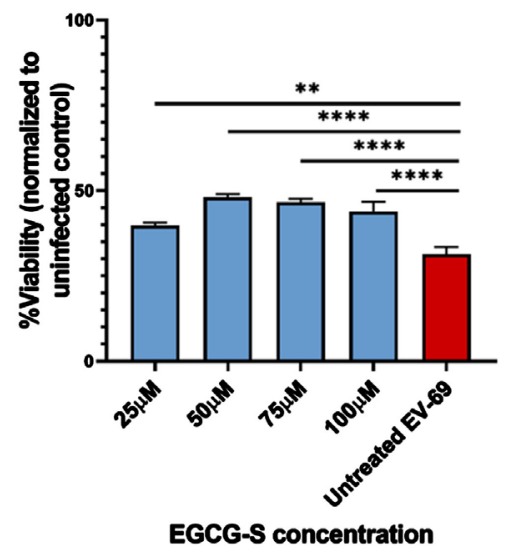

(a)

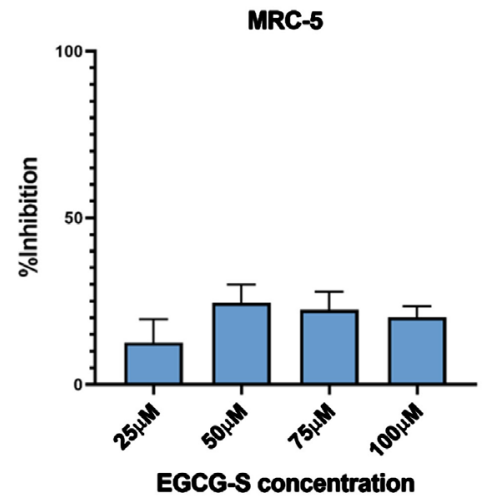

(b)

Figure 2. EV-69 mediated cell death and mitochondrial dysfunction is moderately inhibited in A549 cells and MRC-5 cells after EGCG-S treatment. A549 cells (5 replicates) and MRC- 5 cells ( 3 replicates) were infected with untreated or with $25,50,75$, or $100 \mu \mathrm{M}$ EGCG-S treated virus for $1 \mathrm{hr}$ and cell viability was quantified $24 \mathrm{hrs}$ later using the CellTiter 96Aqueous One Solution Cell Proliferation Assay (MTS) (Promega). A549 cells and MRC- 5 cells had higher viability compared to untreated infected cells 24 hrs later (a). The $\%$ Inhibition calculated based on these viabilities was lower than $50 \%$ for both cell lines (b). Data are presented as mean \pm SEM. Statistical analysis was performed for the viability assay using a one-way Anova with Dunnett's post-hoc test comparing results to the untreated EV-69 infected control, ${ }^{*} \mathrm{p}<0.5,{ }^{* *} \mathrm{p}<0.01$, ${ }^{* *} \mathrm{p}<0.001$, ${ }^{* * *} \mathrm{p}<0.0001$, ns = non-significant.

\subsection{Viral ToxGlo ATP Detection Assay}

To gain insight into the metabolic activity of the MRC-5 cells for which EGCG-S mediated inhibition of infection was not prominent, we also assayed for ATP levels as an indicator of mitochondrial function and cell viability. Treatment with EGCG-S alone did not negatively impact ATP production in these cells, as measured through the ToxGlo ATP detection assay (Figure 3(a)). Treatment of MRC-5 with EGCG-S did not influence ATP production, therefore treatment with EGCG-S had no negative impact on cell viability. There is no statistical difference between untreated MRC-5 cells and cells treated with EGCG-S up to $100 \mu \mathrm{M}$ 


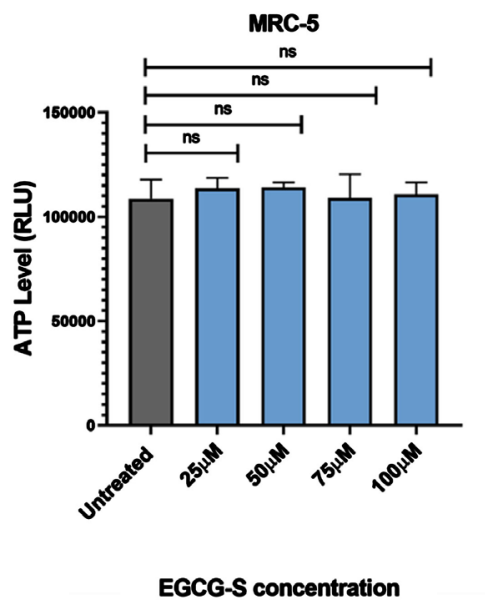

(a)

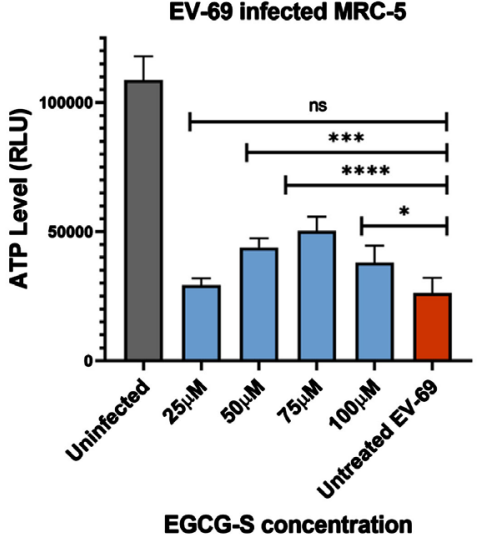

(b)

Figure 3. EGCG-S treatment increased ATP production in infected MRC-5 cells. MRC-5 cells were infected with $25,50,75$, or $100 \mu \mathrm{M}$ EGCG-S treated virus for $1 \mathrm{~h}$ and ATP levels were assayed $24 \mathrm{~h}$ later using the Viral ToxGlo ${ }^{\mathrm{rm}}$ assay kit (Promega Corp., Madison, WI). Treatment of MRC-5 with EGCG-S did not influence ATP production (A). Infected MRC-5 cells with treated or untreated EV-69 resulted in moderately increased intracellular ATP relative to the untreated EV-69 in MRC-5 cells (B). Data are presented as mean \pm SD from one experiment (5 replicates), ${ }^{*} \mathrm{p}<0.5,{ }^{* *} \mathrm{p}<0.01,{ }^{* *} \mathrm{p}<0.001,{ }^{* * *} \mathrm{p}<0.0001$, ns = non-significant.

(Figure 3(a)). However, the limited efficacy of EGCG-S in inhibiting infection was further observed when examining the ATP levels of infected MRC- 5 cells. Intracellular ATP for these cells was increased with EGCG-S treatment but was still substantially lower than the uninfected control (Figure 3(b)). This suggests that EV-69 infection still greatly compromised MRC-5 cells, and EGCG-S treatment only moderately protects MRC- 5 cells from mitochondrial dysfunction as well loss of metabolic activity induced by EV-69 infection.

\subsection{ROS Detection Assay}

Treatment with EGCG-S has thus far been demonstrated to reduce mitochondrial dysfunction, and overall cytotoxicity caused by EV-69 infection. These effects are known to occur in enterovirus infection as a result of oxidative stress [38] [39] [40]. While MRC-5 cells are highly susceptible to EV-69 mediated infection, EGCG-S treatment moderately increased cell viability in comparison to untreated controls (Figure 2(a) and Figure 2(b)). EGCG has been reported to modulate cellular redox that is associated with inhibition of infection [26] [29] [30] [31]. Therefore, this study investigated the antioxidant potential of EGCG-S in inhibiting EV-69 infection. To assess whether the protective effects of EGCG-S observed may be due to its antioxidant potential, the total intracellular level of a major reactive oxygen species (ROS), hydrogen peroxide $\left(\mathrm{H}_{2} \mathrm{O}_{2}\right)$ was further examined. EGCG-S treatment resulted in a statistically significant decrease in the total $\mathrm{H}_{2} \mathrm{O}_{2}$ levels following 25 - $75 \mu \mathrm{M}$ treatments in comparison to the untreated EV-69 infected control (Figure 4). Conversely, treatment at 100 


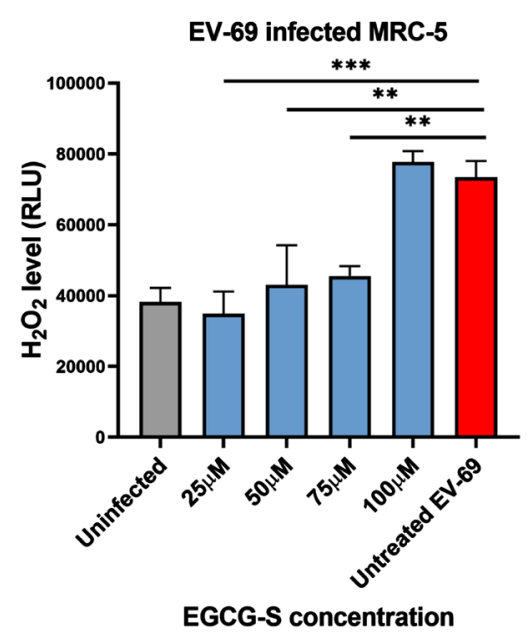

Figure 4. ROS generation is decreased in MRC-5 cells infected with EGCG-S treated EV-69. MRC-5 cells were infected with untreated or 25, 50, 75, or $100 \mu \mathrm{M}$ EGCG-S treated virus for $1 \mathrm{~h}$ and $\mathrm{H}_{2} \mathrm{O}_{2}$ levels were assayed $12 \mathrm{~h}$ later using the ROS-Glo ${ }^{\mathrm{TM}} \mathrm{H}_{2} \mathrm{O}_{2}$ assay kit (Promega Corp., Madison, WI). MRC-5 cells had lower overall $\mathrm{H}_{2} \mathrm{O}_{2}$ levels when infected with 25 - 75 MM EGCG-S treated EV-69 in comparison to the untreated EV-69 control. Data are presented as mean \pm SEM from two independent experiments with two and four replicates, respectively. Statistical analysis was performed using a one-way Anova with Dunnett's post-hoc test comparing results to the untreated EV-69 infected control, ${ }^{* *} \mathrm{p}<0.01,{ }^{* * *} \mathrm{p}<0.001$.

$\mu \mathrm{M}$ resulted in greater $\mathrm{H}_{2} \mathrm{O}_{2}$ levels than in uninfected cells. This may be a result of EGCG-S mediated cytotoxicity in addition to EV-69 infection, as EGCG may produce $\mathrm{H}_{2} \mathrm{O}_{2}$ in culture at concentrations above $50 \mu \mathrm{M}$ [41] [42] [43]. This suggests the protective effects of EGCG-S may be due to its antioxidant activity, at concentrations below $100 \mu \mathrm{M}$. While a difference in $\mathrm{H}_{2} \mathrm{O}_{2}$ levels were observed in this infection assay, the effect of EGCG-S on total intracellular ROS needs to be investigated to further understand if the antiviral effect of EGCG-S may be mediated by its ability to scavenge radicals that result in cell damage caused by total ROS collectively.

\subsection{Microscopic Observation of EV-69 Infected MRC-5 Cells and EGCG-S Treated Infected MRC-5 Cells}

Infection with EV-69 causes a decrease in viability, characterized as more than $90 \%$ of cells exhibiting rounding cytopathic effects or by the presence of cellular debris as a result of prominent cell lysis at $48 \mathrm{hrs}$ post infection (hpi). These cytopathic effects (CPE) were investigated in MRC- 5 cells infected with untreated EV-69 or EV-69 that was pretreated for $1 \mathrm{hr}$ with EGCG-S ( 25 to $100 \mu \mathrm{M})$ before infecting cells. As a result of EGCG-S treatment of EV-69, more cell proliferation was observed for MRC- 5 cells, particularly at 50 and $75 \mu \mathrm{M}$, than for the untreated EV-69 infected control (Figure 5). Cytotoxicity became apparent at 100 $\mu \mathrm{M}$ for MRC- 5 cells, however, as demonstrated by increased rounding and debris relative to cells infected with untreated EV-69 (Figure 5). These morphological changes may be a result of both viral infection and toxicity of EGCG-S. 


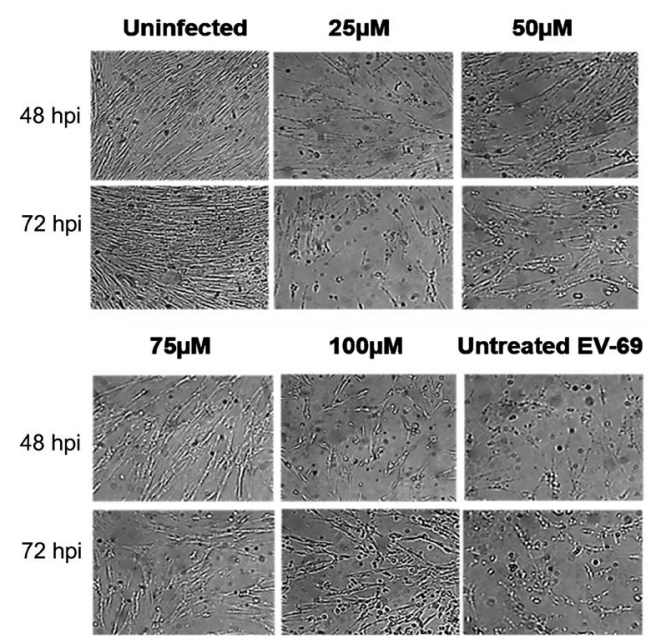

Figure 5. EGCG-S protects against EV-69 induced CPE in MRC-5 cells. MRC-5 cells were infected with EV-69 treated with 25, 50, 75, or $100 \mu \mathrm{M}$ EGCG-S then incubated for 48 hrs or 72 hrs before cell morphology was examined via microscopy. MRC- 5 cells infected with 50 or $75 \mu$ M EGCG-S pre-treated EV-69 had reduced CPE 48 and 72 hrs post infection (hpi) as compared to MRC-5 infected with untreated EV-69.

Thus, microscopic observation indicated that EGCG-S reduces EV-69 induced cytopathic effects. Altogether, this supports that EGCG-S can prevent EV-69 mediated damage to cells but at concentrations lower than $100 \mu \mathrm{M}$.

\section{Discussion}

The benefits of EGCG-S treatment in viral infections with greatly differing clinical manifestations have been extensively investigated. In this study, we assess the antiviral effect of EGCG-S on EV-69, an enterovirus that was observed to cause productive infection in respiratory epithelial and fibroblast cells in our study. Following treatment with EGCG-S, MRC-5 cells had reduced EV-69 mediated cytotoxicity in comparison to the untreated virus control, albeit with low percent of inhibition of infection. In contrast, other studies reported EGCG-S mediated inhibition of infection to be much higher for HSV-1 infected Vero cells and A549 cells [23] [33]. This difference in efficacy of EGCG-S observed in our study may be attributed to the structural differences in viruses treated, as this modified form of EGCG is postulated to be more efficacious than unmodified EGCG due to having better affinity for the viral envelope and EV-69 is a non-enveloped virus [23]. Furthermore, it is possible that the modes of action of EGCG-S against cells infected with RNA versus DNA viruses also differ. Moreover, we observed that levels of hydrogen peroxide were reduced in MRC- 5 cells infected with EGCG-S treated virus at concentrations lower than $100 \mu \mathrm{M}$, suggesting EV-69 mediated oxidative stress was inhibited. Determining the causes of the moderate antiviral activity observed in this study requires understanding of both EV-69 pathogenesis as well as the full range of mechanisms in which EGCG-S inhibits viral infections.

The proposed antiviral mechanism(s) of EGCG has differed among studies. 
Researchers demonstrated that it is likely due to EGCG competing with sialic acid or heparin sulfate containing host cell receptors for viral attachment. This finding is supported by reduced antiviral efficacy against poliovirus. This reduction was associated with poliovirus not being dependent on sialic acid or heparin sulfate containing receptors for entry [21]. However, a different study attributed EGCG-mediated inhibition of EV-71 replication to a reduction of EV-71 induced oxidative stress [26]. Our study demonstrated that hydrogen peroxide was reduced with EGCG-S treatment. EV-69 was treated with EGCG-S prior to infection, which would allow attachment of EGCG-S to the virus. Thus, it is possible that inhibition of EV-69 infection may have occurred through both modulation of cellular redox and prevention of virus attachment to cells. Subsequent research in our laboratory found that EGCG-S inhibited attachment and penetration of HSV-2 in cultured Vero cells (unpublished data). These reports suggest that there may be multiple mechanisms whereby EGCG-S inhibits infection, depending on different virus specific factors that cause cytotoxicity.

While EGCG-S has been formulated as a topical cream for treatment of HSV-1 [23] [44] its clinical application for treatment of other viral diseases is at the preliminary stages. Murine models of Coxsackie B3 and influenza A virus infection supported the bioavailability of EGCG in vivo with oral administration, as treatment of mice resulted in improved survival correlated with reduced viral replication [25] [45] [46] [47]. Our study suggests that EGCG-S reduced the oxidative damage caused by EV-69 infection and increased the viability of infected cells, thus potentially reducing viral replication. While EGCG was shown to reduce viral replication in previous studies, contrasting effects on the inflammatory response associated with viral infections were reported. For influenza A infection, antiviral effects were associated with reduced lung inflammation, in agreement with studies reporting the overall anti-inflammatory effect of EGCG [48] [49] [50]. On the other hand for Coxsackie B3 infection, EGCG treatment was found to greatly inhibit viral replication in heart tissues of mice and associated myocarditis but did not decrease pro-inflammatory cytokine levels [25]. Dampening of the pro-inflammatory cytokine response may be beneficial for viral diseases that cause severe symptoms in result of a cytokine storm, such as influenza A virus or the newly emerged SARS-COV-2 [51], but inhibition of inflammation in other viral infections may lead to prolonged disease or persistent infection due to the suboptimal immune response necessary for clearance of infection. The role of EGCG-S in inhibiting infection will thus require closer examination of the antiviral immune response, including the production of type I interferons (IFNs), in untreated versus treated infected cells. Examination of the cytokine milieu as well as antigen presenting cell chemotaxis and targeting of EGCG-S treated infected cells would help elucidate whether EGCG-S treatment facilitates clearance of enterovirus infection or enables persistent infection due to interference with the antiviral response.

Furthermore, as enterovirus infections may cause severe disease progression to neurological complications, the ability of EGCG-S to serve as an antiviral in 
early versus later stages of infection will need to be assessed. Future studies investigating the application of EGCG-S 24 or $48 \mathrm{hrs}$ following infection can help characterize the efficacy of EGCG-S in inhibiting EV-69 infection at different stages, as well as further distinguish the role of EGCG-S in virus attachment. Thus far, our data suggest that EV-69 mediated cytotoxicity can be reduced with EGCG-S application in the early stages of infection and merit further investigation into maximization of efficacy alone or in combinatorial strategies. If applicable to enteroviral infections in vivo, EGCG-S can contribute to the prevention of disease progression and spread in areas in which enterovirus outbreaks occur.

\section{Acknowledgements}

We would like to thank Dr. A. DiLorenzo in the Biology Department at Montclair State University for providing us with MRC-5 cells and an ROS-Glo assay kit for $\mathrm{H}_{2} \mathrm{O}_{2}$ measurement. We would also like to acknowledge Dr. J. Siekierka in the Chemistry and Biochemistry Department at Montclair State University for providing us his facility to conduct luminescence-based experiments. This research was funded in part by the Faculty Scholarship Program at Montclair State University.

\section{Authors' Contributions}

HM, LHL, and SDA designed the study. SDA supervised HM in the laboratory. HM and SDA conducted the experiments. HM performed all statistical analyses. HM, LHL, and SDA drafted the manuscript. All authors read and approved the final manuscript.

\section{Conflicts of Interest}

The authors declare no conflicts of interest.

\section{References}

[1] Chen, B.S., Lee, H.C., Lee, K.M., Gong, Y.N. and Shih, S.R. (2020) Enterovirus and Encephalitis. Frontiers in Microbiology, 11, 261. https://doi.org/10.3389/fmicb.2020.00261

[2] Royston, L. and Tapparel, C. (2016) Rhinoviruses and Respiratory Enteroviruses: Not as Simple as $A B C$. Viruses, 8, 16. https://doi.org/10.3390/v8010016

[3] Romero, J.R. and Kimberlin, D.W. (2003) Molecular Diagnosis of Viral Infections of the Central Nervous System. Clinics in Laboratory Medicine, 23, 843-865. https://doi.org/10.1016/S0272-2712(03)00084-2

[4] Huang, W., Wang, G., Zhuge, J., Nolan, S.M., Dimitrova, N. and Fallon, J.T. (2015) Whole-Genome Sequence Analysis Reveals the Enterovirus D68 Isolates during the United States 2014 Outbreak Mainly Belong to a Novel Clade. Scientific Reports, 5 , Article No. 15223. https://doi.org/10.1038/srep15223

[5] Kim, M.S. and Racaniello, V.R. (2007) Enterovirus 70 Receptor Utilization Is Controlled by Capsid Residues that Also Regulate Host Range and Cytopathogenicity. Journal of Virology, 81, 8648-8655. https://doi.org/10.1128/JVI.01569-06 
[6] Gilbert, G.L., Dickson, K.E., Waters, M.J., Kennett, M.L., Land, S.A. and Sneddon, M. (1988) Outbreak of Enterovirus 71 Infection in Victoria, Australia, with a High Incidence of Neurologic Involvement. The Pediatric Infectious Disease Journal, 7, 484-487. https://doi.org/10.1097/00006454-198807000-00007

[7] Rosenfeld, A.B., Warren, A.L. and Racaniello, V.R. (2019) Neurotropism of Enterovirus D68 Isolates Is Independent of Sialic Acid and Is Not a Recently Acquired Phenotype. Host-Microbe Biology, 10, e2370-19.

https://doi.org/10.1128/mBio.02370-19

[8] Wadia, N.H., Wadia, P.N., Katrak, S.M. and Misra, V.P. (1983) A Study of the Neurological Disorder Associated with Acute Haemorrhagic Conjunctivitis Due to Enterovirus 70. Journal of Neurology, Neurosurgery, and Psychiatry, 46, 599-610. https://doi.org/10.1136/jnnp.46.7.599

[9] Thong, W.Y., Han, A., Wang, S.J.F., Lin, J., Isa, M.S., Koay, E.S.C., et al. (2017) Enterovirus Infections in Singaporean Children: An Assessment of Neurological Manifestations and Clinical Outcomes. Singapore Medical Journal, 58, 189-195. https://doi.org/10.11622/smedj.2016099

[10] Rosen, L., Schmidt, N.J. and Kern, J. (1973) Toluca-1, a Newly Recognized Enterovirus. Archives of Virology, 40, 132-136. https://doi.org/10.1007/BF01242645

[11] Yin-Murphy, M. and Almond, J.W. (1996) Picornaviruses. In: Baron, S., Ed., Chapter 53 Medical Microbiology, 4th Edition, University of Texas Medical Branch at Galveston, Galveston.

[12] Fernandez-Garcia, M.D., Majumdar, M., Kebe, O., Ndiaye, K. and Martin, J. (2018) Identification and Whole-Genome Characterization of a Recombinant Enterovirus B69 Isolated from a Patient with Acute Flaccid Paralysis in Niger, 2015. Scientific Reports, 8, Article No. 2181. https://doi.org/10.1038/s41598-018-20346-9

[13] Bessaud, M., Pillet, S., Ibrahim, W., Joffret, M.L., Pozzetto, B., Delpeyroux, F., et al. (2012) Molecular Characterization of Human Enteroviruses in the Central African Republic: Uncovering Wide Diversity and Identification of a New Human Enterovirus A71 Genogroup. Journal of Clinical Microbiology, 50, 1650-1658. https://doi.org/10.1128/JCM.06657-11

[14] Sadeuh-Mba, S.A., Bessaud, M., Massenet, D., Joffret, M.L., Endegue, M.C., Njouom, R., et al. (2013) High Frequency and Diversity of Species C Enteroviruses in Cameroon and Neighboring Countries. Journal of Clinical Microbiology, 51, 759-770. https://doi.org/10.1128/JCM.02119-12

[15] Rao, C.D., Yergolkar, P. and Shankarappa, K.S. (2012) Antigenic Diversity of Enteroviruses Associated with Nonpolio Acute Flaccid Paralysis, India, 2007-2009. Emerging Infectious Diseases, 18, 1833-1840. https://doi.org/10.3201/eid1811.111457

[16] Oyero, O.G., Adu, F.D. and Ayukekbong, J.A. (2014) Molecular Characterization of Diverse Species Enterovirus-B Types from Children with Acute Flaccid Paralysis and Asymptomatic Children in Nigeria. Virus Research, 189, 189-193. https://doi.org/10.1016/j.virusres.2014.05.029

[17] Laxmivandana, R., Yergolkar, P., Gopalkrishna, V. and Chitambar, S.D. (2013) Characterization of the Non-Polio Enterovirus Infections Associated with Acute Flaccid Paralysis in South-Western India. PLoS ONE, 8, e61650. https://doi.org/10.1371/journal.pone.0061650

[18] Singh, D.V., Kumar, A., Kumar, P., Baluni, M., Ghildiyal, S., Kumar, R., et al. (2016) An Outbreak of Encephalitis Associated with Echovirus 19 in Uttar Pradesh, India, in 2011. Archives of Virology, 161, 967-970.

https://doi.org/10.1007/s00705-015-2714-6 
[19] Melok, A.L., Lee, L.H., Mohamed, Y.S.A. and Chu, T. (2018) Green Tea Polyphenol Epigallocatechin-3-Gallate-Stearate Inhibits the Growth of Streptococcus mutans. A Promising New Approach in Caries Prevention. Dentistry Journal(Basel), 6, 38. https://doi.org/10.20944/preprints201807.0138.v1

[20] Inacio, J.D.F., Gervazoni, L., Canto-Cavalheiro, M.M. and Almeida-Amaral E.E. (2014) The Effect of (-)-Epigallocatechin 3-O-Gallate in Vitro and in Vivo in Leishmania braziliensis. Involvement of Reactive Oxygen Species as a Mechanism of Action. PLoS Neglected Tropical Diseases, 8, e3093.

https://doi.org/10.1371/journal.pntd.0003093

[21] Colpitts, C.C. and Schang, L.M. (2014) A Small Molecule Inhibits Virion Attachment to Heparan Sulfate- or Sialic Acid-Containing Glycans. Journal of Virology. 88, 7806-7817. https://doi.org/10.1128/JVI.00896-14

[22] Chu, C., Deng, J., Man, Y. and Qu, Y. (2017) Green Tea Extracts Epigallocatechin-3-Gallate for Different Treatments. Biomed Research International, 2017, Article ID: 5615647. https://doi.org/10.1155/2017/5615647

[23] de Oliveira, A., Adams, S.D., Lee, L.H., Murray, S.R., Hsu, S.D., Hammond, J.R., et al. (2013) Inhibition of Herpes Simplex Virus Type 1 with the Modified Green Tea Polyphenol Palmitoyl-Epigallocatechin Gallate. Food and Chemical Toxicology, 52, 207-215. https://doi.org/10.1016/j.fct.2012.11.006

[24] Xu, J., Xu, Z. and Zheng, W. (2017) A Review of the Antiviral Role of Green Tea Catechins. Molecules. 22, Article ID: 1337. https://doi.org/10.3390/molecules22081337

[25] He, X., Gao, B., Zhou, L. and Xiong, S. (2017) Green Tea Polyphenol Epigallocatechin-3-Gallate-Alleviated Coxsackievirus B3-Induced Myocarditis through Inhibiting Viral Replication but Not through Inhibiting Inflammatory Responses. Journal of Cardiovascular Pharmacology, 69, 41-47. https://doi.org/10.1097/FJC.0000000000000439

[26] Ho, H.Y., Cheng, M.L., Weng, S.F., Leu, Y.L. and Chiu, D.T. (2009) Antiviral Effect of Epigallocatechin Gallate on Enterovirus 71. Journal of Agricultural and Food Chemistry, 57, 6140-6147. https://doi.org/10.1021/jf901128u

[27] Giunta, B., Obregon, D., Hou, H., Zeng, J., Sun, N., Nikolic, V., et al. (2006) EGCG Mitigates Neurotoxicity Mediated by HIV-1 Proteins gp120 and Tat in the Presence of IFN- $\gamma$ : Role of JAK/STAT1 Signaling and Implications for HIV-Associated Dementia. Brain Research, 1123, 216-225.

https://doi.org/10.1016/j.brainres.2006.09.057

[28] Kaihatsu, K., Yamabe, M. and Ebara, Y. (2018) Antiviral Mechanism of Action of Epigallocatechin-3-O-Gallate and Its Fatty Acid Esters. Molecules, 23, Article ID: 2475. https://doi.org/10.3390/molecules23102475

[29] Steinmann, J., Buer, J., Pietschmann, T. and Steinmann, E. (2013) Anti-Infective Properties of Epigallocatechin-3-Gallate (EGCG), a Component of Green Tea. British Journal of Pharmacology, 168, 1059-1073. https://doi.org/10.1111/bph.12009

[30] Yang, X.Q., Shen, S.R., Hou, J.W., Zhao, B.L. and Xin, W.J. (1994) Mechanism of Scavenging Effects of (-)-Epigallocatechin Gallate on Active Oxygen Free Radicals. Acta Pharmacologica Sinica, 15, 350-353.

[31] Zhang, H.S., Wu, T.C., Sang, W.W. and Ruan, Z. (2012) EGCG Inhibits Tat-Induced LTR Transactivation: Role of Nrf2, AKT, AMPK Signaling Pathway. Life Sciences, 90, 747-754. https://doi.org/10.1016/j.lfs.2012.03.013

[32] Zhong, Y. and Shahidi, F. (2011) Lipophilized Epigallocatechin Gallate (EGCG) De- 
rivatives as Novel Antioxidants. Journal of Agricultural and Food Chemistry, 59, 6526-6533. https://doi.org/10.1021/jf201050j

[33] Patel, S.N., Adams, S.D. and Lee L.H. (2018) Inhibition of Herpes Simplex Virus-1 by the Modified Green Tea Polyphenol EGCG-Stearate. Advances in Bioscience and Biotechnology, 9, 679-690. https://doi.org/10.4236/abb.2018.912046

[34] Hellferscee, O., Tempia, S., Walaza, S., Variava, E., Dawood, H., et al. (2017) Enterovirus Genotypes among Patients with Severe Acute Respiratory Illness, Influenza-Like Illness, and Asymptomatic Individuals in South Africa, 2012-2014. Journal of Medical Virology, 89, 1759-1767. https://doi.org/10.1002/jmv.24869

[35] Lin, J.Y. and Shih, S.R. (2014) Cell and Tissue Tropism of Enterovirus 71 and Other Enteroviruses Infections. Journal of Biomedical Science, 21, 18.

https://doi.org/10.1186/1423-0127-21-18

[36] Chonmaitree, T., Ford, C., Sanders, C. and Lucia, H.L. (1988) Comparison of Cell Cultures for Rapid Isolation of Enteroviruses. Journal of Clinical Microbiology, 26, 2576-2580. https://doi.org/10.1128/JCM.26.12.2576-2580.1988

[37] Flores, D.J., Lee, L.H. and Adams, S.D. (2016) Inhibition of Curcumin-Treated Herpes Simplex Virus 1 and 2 in Vero Cells. Advances in Microbiology, 6, 276-287. https://doi.org/10.4236/aim.2016.64027

[38] Bai, Z., Zhao, X., Li, C., Sheng, C. and Li, H. (2020) EV71 Virus Reduces Nrf2 Activation to Promote Production of Reactive Oxygen Species in Infected Cells. Gut Pathogens. 12, Article No. 22. https://doi.org/10.1186/s13099-020-00361-W

[39] You, L., Chen, J., Liu, W., Xiang, Q., Luo, Z., Wang, W., et al. (2020) Enterovirus 71 Induces Neural Cell Apoptosis and Autophagy through Promoting ACOX1 Downregulation and ROS Generation. Virulence, 11, 537-553. https://doi.org/10.1080/21505594.2020.1766790

[40] Cheng, M.L., Weng, S.F., Kuo, C.H. and Ho, H.Y. (2014) Enterovirus 71 Induces Mitochondrial Reactive Oxygen Species Generation that Is Required for Efficient Replication. PLoS ONE, 9, e113234. https://doi.org/10.1371/journal.pone.0113234

[41] Kim, H.S., Quon, M.J. and Kim, J.A. (2014) New Insights into the Mechanisms of Polyphenols beyond Antioxidant Properties; Lessons from the Green Tea Polyphenol, Epigallocatechin 3-Gallate. Redox Biology, 2, 187-195.

https://doi.org/10.1016/j.redox.2013.12.022

[42] Nakagawa, H., Hasumi, K., Woo, J.T., Nagai, K. and Wachi, M. (2004) Generation of Hydrogen Peroxide Primarily Contributes to the Induction of $\mathrm{Fe}(\mathrm{II})$-Dependent Apoptosis in Jurkat Cells by (-)-Epigallocatechin Gallate. Carcinogenesis, 25, 1567-1574. https://doi.org/10.1093/carcin/bgh168

[43] Nakagawa, H., Wachi, M., Woo, J.T., Kato, M., Kasai, S., et al. (2002) Fenton Reaction Is Primarily Involved in a Mechanism of (-)-Epigallocatechin-3-Gallate to Induce Osteoclastic Cell Death. Biochemical Biophysical Research Communications, 292, 94-101. https://doi.org/10.1006/bbrc.2002.6622

[44] Zhao, M., Jiang, J., Zheng, R., Pearl, H., Dickinson, D., Fu, B., et al. (2012) A Proprietary Topical Preparation Containing EGCG-Stearate and Glycerin with Inhibitory Effects on Herpes Simplex Virus: Case Study. Inflammation and Allergy-Drug Targets, 11, 364-368. https://doi.org/10.2174/187152812803251033

[45] Xiao, X., Yang, Z.Q., Shi, L.Q., Liu, J. and Chen, W. (2008) Antiviral Effect of Epigallocatechin Gallate (EGCG) on Influenza a Virus. China Journal of Chinese Materia Medica, 33, 2678-2682.

[46] Ling, J.X., Wei, F., Li, N., Li, J.L., Chen, L.J., Liu, Y.Y., et al. (2012) Amelioration of 
Influenza Virus-Induced Reactive Oxygen Species Formation by Epigallocatechin Gallate Derived from Green Tea. Acta Pharmacologica Sinica, 33, 1533-1541. https://doi.org/10.1038/aps.2012.80

[47] Onishi, S., Kitazawa, H., Meguro, S. and Tokimitsu, I. (2018) Green Tea Extracts Reduce Leukocyte Cell-Derived Chemotaxin 2 and Selenoprotein P Levels in the Livers of C57BL/6J Mice Fed a High-Fat Diet. Bioscience, Biotechnology, and Biochemistry, 82, 1568-1575. https://doi.org/10.1080/09168451.2018.1480349

[48] Haghighatdoost, F. and Hariri, M. (2019) The Effect of Green Tea on Inflammatory Mediators: A Systematic Review and Meta-Analysis of Randomized Clinical Trials. Phytotherapy Research, 33, 2274-2287. https://doi.org/10.1002/ptr.6432

[49] Liu, Q., Qian, Y., Chen, F., Chen, X., Chen, Z. and Zheng, M. (2014) EGCG Attenuates Pro-Inflammatory Cytokines and Chemokines Production in LPS-Stimulated L02 Hepatocyte. Acta Biochimica et Biophysica Sinica, 46, 31-39. https://doi.org/10.1093/abbs/gmt128

[50] Melgarejo, E., Medina, M.A., Sanchez-Jimenez, F., and Urdiales, J.L. (2009) Epigallocatechin Gallate Reduces Human Monocyte Mobility and Adhesion in Vitro. British Journal of Pharmacology, 158, 1705-1712. https://doi.org/10.1111/j.1476-5381.2009.00452.x

[51] Ragab, D., Salah, Eldin, H., Taeimah, M., Khattab, R. and Salem, R. (2020) The COVID-19 Cytokine Storm; What We Know So Far. Frontiers in Immunology, 11, 1446. https://doi.org/10.3389/fimmu.2020.01446 